\title{
Influence of the Hippo-YAP signalling pathway on tumor associated macrophages (TAMs) and its implications on cancer immunosuppressive microenvironment
}

\author{
Wenjie Yang, Shikun Yang, Feng Zhang, Feng Cheng, Xuehao Wang, Jianhua Rao \\ Hepatobiliary Center, The First Affiliated Hospital of Nanjing Medical University, Key Laboratory of Liver Transplantation, Chinese Academy of \\ Medical Sciences, NHC Key Laboratory of Living Donor Liver Transplantation, Nanjing 210029, China \\ Contributions: (I) Conception and design: J Rao, W Yang; (II) Administrative support: X Wang; (III) Provision of study materials or patients: W Yang, \\ S Yang, F Zhang, F Cheng; (IV) Collection and assembly of data: W Yang, F Zhang; (V) Data analysis and interpretation: J Rao; (VI) Manuscript \\ writing: All authors; (VII) Final approval of manuscript: All authors. \\ Correspondence to: Jianhua Rao. Hepatobiliary Center, The First Affiliated Hospital of Nanjing Medical University, Key Laboratory of Liver \\ Transplantation, Chinese Academy of Medical Sciences, Nanjing 210029, China. Email: raojh@njmu.edu.cn.
}

\begin{abstract}
A large number of immune cells are present in the tumour microenvironment (TME), of which, tumour-associated macrophages (TAMs) are among the most important and highly infiltrated cells, and mainly include the M1 type classically activated and M2 type alternatively activated TAMs. Both cell types are known to play an important role in tumour initiation and proliferation. It has recently been confirmed that the TAMs in tumours tend to be dominated by the M2 type. However, the precise mechanism underlying TAM recruitment and polarization in the immune microenvironment remains to be elucidated. The Hippo-Yes-associated protein (YAP) signalling pathway is one of the most extensively discussed mechanism for the regulation of tumour proliferation, migration, angiogenesis, and invasion in recent years. To date, several studies have revealed that YAP is involved in the interrelating interactions between tumour and immune cells, particularly the TAMs. In this review, we have summarized the mechanism by which the YAP regulates the activity of TAMs and its impact on the TME.
\end{abstract}

Keywords: Hippo-YAP signalling pathway; tumour associated macrophages; tumour microenvironment (TME); tumour immunosuppression

Submitted Nov 16, 2019. Accepted for publication Jan 14, 2020.

doi: $10.21037 /$ atm.2020.02.11

View this article at: http://dx.doi.org/10.21037/atm.2020.02.11

\section{Introduction}

The Hippo signalling pathway is a newly discovered tumour suppressor signal pathway known to regulate organ size. It was first identified in Drosophila melanogaster, and is composed of one oncogene and multiple tumour suppression genes. Yes-associated protein (YAP) acts as a crucial transcription factor in promoting tumour formation and development, and is responsible for the expression of several pro-oncogenes such as CTGF, KRAS and Wnt/ $\beta$-catenin (1-5). In mammals, the analogue of Hippo is MST1/2, a serine/threonine kinase upstream of YAP. In humans, Hippo-YAP signalling pathway activation accompanied by the MST1/2 kinase phosphorylation and LAST1/2 activation, followed by the phosphorylation of the corresponding YAP sites and WW domaincontaining transcription regulator 1 (TAZ), which are two transcriptional co-activating factors, limit their entry into the nucleus, retain them in the cytoplasm. YAP lacks the DNA binding domain. However, being a transcriptional coactivator, it binds to member of the transcription enhanced associated domain (TEAD) family, which acts as it DNA-binding domains. Recently, it has been reported that the 14-3-3 protein can induce the YAP phosphorylation, causing it to be detained in the cytoplasm. Additionally, 
YAP phosphorylation leads to the recruitment of the E3 ubiquitin ligase SCF to induce its ubiquitination and proteasome degradation (6-11).

TAMs have long been considered as one of the most important immune cells in the tumour microenvironment (TME) and play a significant role in conferring innate and adaptive immunity to the host. Based on the surrounding conditions of the immune microenvironment, TAMs can be polarized into those of the classically activated M1 and alternatively activated M2 phenotypes $(12,13)$ (Figure 1). It is now known that the M1 type TAMSs secrete large amounts of inflammatory factors such as interleukin (IL)1 and tumour necrosis factor- $\alpha(\mathrm{TNF}-\alpha)$, which promote the expression of inducible nitric oxide synthase (INOS) and enhance the innate immune response, particularly, by modulating the adaptive immune response through augmenting antigen presentation process. In contrast, the M2 type TAMs mainly secrete IL-10, IL4, arginase-1 (arg-1) and other enzymes or cytokines required to regulate the degree of immune response $(14,15)$. In the TME, macrophages derived from circulation monocytes differentiate into TAMs, which are stimulated by related chemoattractant cytokines. Meanwhile, TAMs are the most abundant population of inflammatory tumour-infiltrating cells, and these TAMs span over various subsets, ranging from the M1 phenotype that promotes inflammation to the alternative M2 phenotype which is involved in resolving inflammation and wound healing $(16,17)$. Owing to the existence of prostaglandin E2 (PGE2) and the transfer growth factor $\beta$ (TGF- $\beta$ ), the Toll-like receptors (TLRs) are not activated in TME $(18,19)$; hence, it is difficult for macrophages to be polarized into the M1 phenotype, which not only promotes tumour invasion and metastasis, but also protects cancerous cells from immune clearance (20-23).

Further studies have identified that Hippo is the major pathway contributing to the immune response in humans, particularly, in functional regulation of TAMs. Being a key effector of the Hippo pathway, YAP not only promotes the development of tumours, but also acts as an immune regulator between immune cells and tumour cells $(24,25)$. Given that TAMs play a non-substitutable role in tumour immunity and exacerbate the overall prognosis, it is important to figure out the regulatory effect of YAP has on them. In this review, we mainly describe the role of the Hippo-YAP signalling pathway as a new target for immunotherapy involving the recruitment of TAMs, their polarization, and tumour immunosuppression, providing new insights into its potential implication against malignancies.

\section{Hippo-YAP signalling pathway regulates TAMs recruitment in tumour tissue}

Numerous studies have reported the recruitment of TAMs strongly associated with poor prognosis in multiple tumour types such as lung, hepatic and colon cancers (26-28). In recent years, it has been widely reported that multiple cytokines and chemokines secreted by tumour cells, such as monocyte chemoattractant protein $1(\mathrm{Mcp}-1)$ and colonystimulating factor-1 (CSF-1) are responsible for the recruitment of TAMs (29-31). Furthermore, an anoxic TEM has been found to be highly correlated with the infiltration of TAMs. For example, structure of the zinc finger E-BOX binding protein 1 (ZEB1) induced by hypoxia has been found to promote the activity of tumour cells secreting CCL8 and induce the epithelial stroma transformation, which is correlated to the infiltration of TAMs (32) (Figure 2). Here, we mainly discuss the functions of YAP during TAM infiltration. YAP activation in tumour cells influences several cytokines and chemokines associated with this process. IL-6 is a well-known pro-inflammatory factor that plays a key role in modulating the immune response and tumour growth, and is considered an effective stimulator for the promotion of monocyte migration (33-35). For example, in brain tumor glioma (or astrocytoma), IL-6 is the major mitogen released from TAMs, which can promote glioma stem cells formation and induce TAMs accumulation in a feed-forward loop. In hepatocellular carcinoma, IL-6 secretion depends on YAP activation partially. Interfering with YAP expression through atorvastatin treatment or employing the YAP-TEAD inhibitor, verteporfin, dramatically inhibits TAMs recruitment in a concentrationdependent manner. In vivo, silencing YAP expression in HepG2 cells can reduce TAMs (with CD206 staining) infiltration in the xenografted tumours. The conditions observed in, Mst1 and Mst2 deficient mice, referred to as $\mathrm{DKO}$, are as follows: spontaneous tumour formation was observed in DKO mice and upregulation of Mcp1 is one of the most pronounced genes among all chemokines. Transcriptome analysis and co-immunofluorescent staining revealed that the population of TAMs in liver is noticeably increased in the livers of DKO mice (36). Moreover, high transcription levels of YAP have been found to be correlated with the formation of tumour initiation cells, following which, they are surrounded by TAMs upon their development. Gou et al. demonstrated that transferring the 


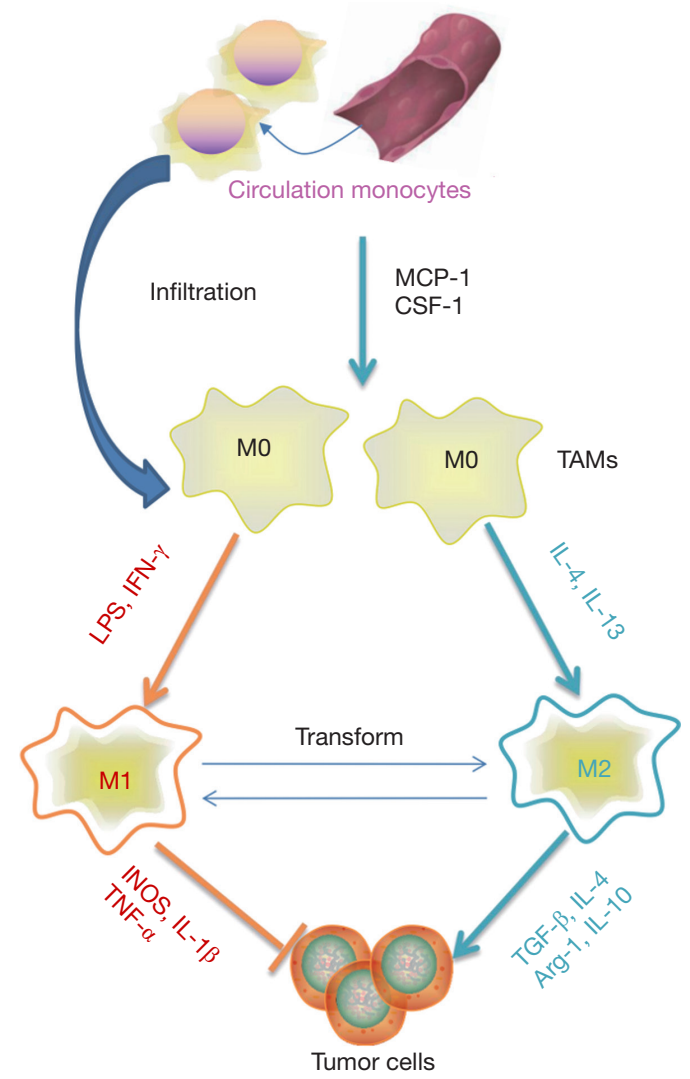

Figure 1 The polarization of tumour associated macrophages: Circulating monocytes can be recruited to tumour microenvironment where they differentiate into TAMs with various phenotypes ranging from the M1 phenotype to the alternative M2 phenotype. This process including multiple classic factors including IL-4, IL-13, LPS and IFN- $\gamma$. M1 phenotype acts as a pro-inflammatory stimuli through secreting large amounts of inflammatory factors like INOS, IL- $1 \beta$ and TNF- $\alpha$. While M2 phenotype releases TGF- $\beta$, IL-4, Arg- 1 and $\mathrm{IL}-10$ to regulate the degree of immune response and boost tumour formation. TAMs, tumour-associated macrophages.

plasmid encoding YAP protein used the Hydrodynamic injection binding transposition technology, which allowed some hepatocytes (approximately $1 \%$ ) to amplify YAP expression stably and induce tumour formation at 4 months, in both the endogenous and exogenous YAP activating tumour mode. Further experiments showed that the overexpression of YAP in tumour initiation cells allows the recruitment of TAMs with high levels of CD206 expression by inducing CCL2 and CSF-1 expression (37). The infiltration of TAMs into the TME is so concerning because it can lead to excessive cross-linking and deposition of TME relevant proteins. The dense stroma offers a relatively safe and exclusive environment for the tumour initiation cells, thereby hindering drug delivery, and promoting tumour growth, metastasis, and drug resistance. Several target preparations and molecules that alter the extracellular matrix surrounding tumour cells by reducing the recruitment of TAMs and reverse this vicious cycle through the Hippo-YAP signalling pathway have been reported (38-40). Tamoxifen can serve as a potential regulator of the immune response in order to manage YAP activity by targeting the $\mathrm{G}$ protein coupled oestrogen receptor (GPER), thus affecting the activation of pancreatic stellate cells (PSCs), ameliorating extracellular matrix remodelling, and reducing the infiltration of TAMs. In pancreatic duct carcinoma, Cortes et al. used fibronectin coated glass to mimic the extracellular matrix and evaluate the migration of TAMs. In the TAMs treated with tamoxifen, it is observed that the area of 1 hour migration had decreased by $30 \%$ compared to that of the control group, suggesting that it inhibits the infiltration of TAMs in the TEM by regulating the adhesion of cells (41). In conclusion, the effect of YAP transcription on the recruitment of TAMs relies mainly on modulating the levels of IL-6, CSF-1, and CCL-2 secreted by the tumour cells, thereby inducing the formation of tumour initiation cells and remodelling the composition of TME. Although the precise mechanism underlying TAM recruitment in the TME and tumour region has still not been completely elucidated, we can claim that YAP is an important participant in this process.

\section{Hippo-YAP signalling pathway regulates TAMs phenotypes}

TME should be considered as a complicated environment in which tumour promoting cytokines and the extracellular matrix structure undergo spontaneous changes. This indicates that the diversity of TAMs in TME is more complex than previous notions. The fate evolution of TAMs is mainly determined by the heterogeneity of undifferentiated circulating monocytes and the microenvironment in which they are located. Activated TAMs can be differentiated into various subgroups, of which M1 and M2 are the two phenotypical polar extremes. Current conceptions show that M1 TAMs possess the ability to kill microorganisms and promote inflammation. In contrast, M2 TAMs can be polarized by IL-4, IL-13 and glucocorticoids to exert an influence on immunosurveillance and boosts tumour maintenance and progression (42-44). In colorectal cancer, an increased 


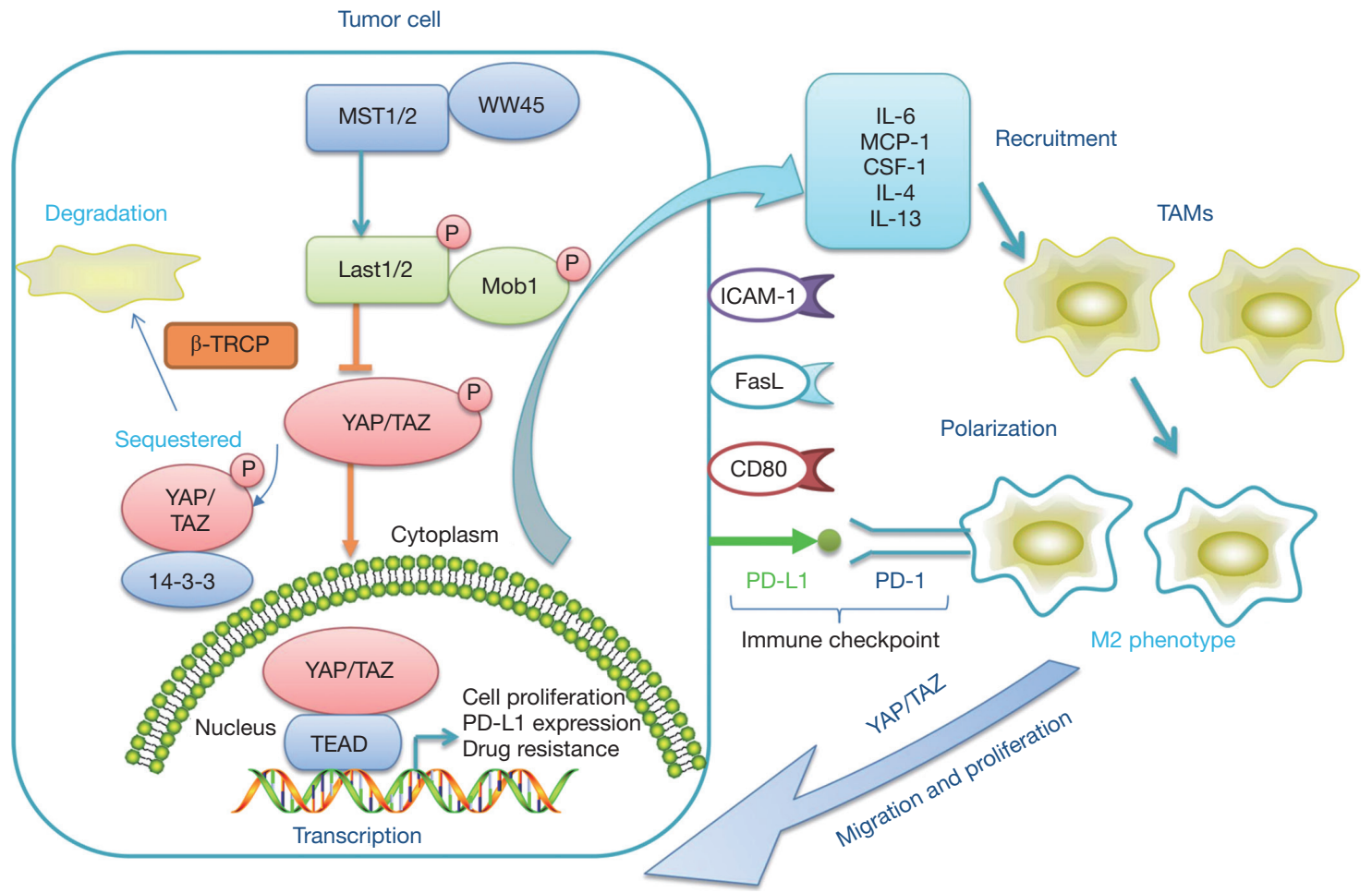

Figure 2 The Hippo signalling pathway in tumour cells and its association with tumour-associated macrophages (TAMs): mammalian sterile 20-like kinases 1 and 2 (MST1/2) combine with the scaffolding protein Salvador/WW45 to phosphorylate the NDR family kinases Lats1 and Lats2 (Lats1/2) downstream, and the adaptor protein Mob1, after which, they phosphorylate the YAP and TAZ. Phosphor-YAP and TAZ would be sustained and degraded in the cytoplasm, thereby preventing nuclear accumulation and leading to cell proliferation, PD-L1 expression, and drug resistance. Furthermore, aberrant YAP transcription in the nucleus is highly correlated with the secretion of IL-6, IL4, MCP-1, CSF-1, and IL-13, which might enable the infiltration of recruited TAMs and induce M2 polarization. The alternatively (M2) activated TAMs are anti-inflammatory cells, which exacerbate the migration and proliferation of tumour cells.

expression of YAP has been shown to be associated with M2 TAM polarization and tumourigenesis through both cell-autonomous and non-autonomous mechanisms. Down-regulation of YAP expression in colon cancer cells inhibits tumour formation, metastasis and generation of cancer stem-like cells, accompanied by a decreased in the levels of cytokines such as IL-4 and IL-13, which is a vital inducer for $\mathrm{M} 2$ polarization generation. In addition to these cell-autonomous mechanism, YAP-silencing in human monocytic cells (THP-1) markedly decreases the levels of M2 markers (ARG1 and CD23), and other polarization related factors, such as $\beta$-catenin, Atk and NF-kB, while no apparent changes are observed in M1 marker (CD64 and CCR7). This phenomenon demonstrates that YAP promotes the polarization of M2 TAMs rather than that of M1 TAMs. It is further explained that the signal pathway promotes M2 polarization through YAP activation in addition to interrelate with M2 inducers (45). Guo et al. also reported that ectopic activation of YAP in hepatocytes induces the formation of liver tumour-initiating cells (TICs) and polarizes peripheral TAMs into M2 phenotype (37). Moreover, in pancreatic ductal adenocarcinoma (PDAC), deletion of YAP shows a significant increase in the proportion of TAMs expressing INOS rather than arginase, suggesting that knockout of YAP promotes the polarization of TAMs into M1 phenotype (46). Additionally, the newly unveiled idea is that a few specific proteins produced by tumour and immune cells during the process of tumour formation are involved in TAMs infiltration and differentiation. Zhang et al. claimed that the extracellular matrix secretory protein, SPON2, promotes the recruitment of M1 TAMs via interacting with integrin $\alpha 4 \beta 1$. Recombinant SPON2 (rSPON2) protein significantly increases the concentration of YAP translocated to the 
nucleus in THP-1 cells, which coexists with M1 phenotype polarization (47). Meanwhile, Kim et al. also showed that in Mst1/2 deficient mice, YAP transcription activity is significantly enhanced in macrophages that exhibits mixed M1 and M2 phenotypes (36). Collectively, both these studies revealed that the polarization of TAMs is related to crosstalk between tumour cells and TAMs through the Mst1/2-Last1/2-YAP pathway. Although cytokines such as IFN- $\gamma$, IL-4 and IL-13 play a clear role in TAMs polarization, the relationship between other stimuli in the TME such as lactate production from glucose, exosomes or free fatty acids, and TAMs phenotype has yet to be completely elucidated (48-50). In a previous study, Xue et al. identified polarization of macrophages at the transcriptional factor level by generating a transcriptomic data set in vitro and vivo. This illustrates that a spectrum of macrophages activation states extend the current $M 1$ versus M2-polarization model. Similarly, others researchers find that in the setting of glioma, TLRs located on TAMs and endogenous ligands released from tumour play a crucial role in TAMs reprogramming with complex surface markings rather than limiting to the M1/M2 classification scheme. The diversity of macrophage polarization may explain the incertitude role of YAP in TAMs phenotypes. In the future, further studies are required to identify the relationship between Yap activation and differential phenotypes of TAMs with complicated stimulus in TME and beyond.

\section{Implications of Hippo-YAP signalling on the immunosuppressive microenvironment}

To date, Hippo-YAP signalling pathway has been characterized not only as a regulator of tumour growth, but also as a crucial inhibitor in cancerous immunity. Tumour immunosuppression is the process that immune system does not recognize or respond to tumour cells which is highly correlated with a decrease in the major histochemical compatibility antigens(MHC-I), deletion of co-stimulatory molecules and various tumour secreting cytokines such as IL-10, TGF- $\beta$, and vascular endothelial growth factor (VEGF). Intensive studies have indicated that YAP transcription levels favor cancerous cells from immune clearance (51-54). Typically, high expression of YAP in the tumour tissue is accompanied by the infiltration of different types of immunosuppressive cells, which aim to maintain immune energy and tolerance, such as the regulatory $\mathrm{T}$ cells (Tregs) and myeloid-derived suppressor cells (MDSC) $(55,56)$ (Figure 3). In this regard, An et al. reported that the formation of invasive lobular carcinoma (ILC) is usually associated with the mutation of CDH1 and PIK3CA. Deletion of $C D H 1$ and activation of PIK3CA leads to tumour formation, which is accompanied by the infiltration of Immunosuppressive cells such as Treg and augments the expression of $P D-L 1$ through a YAP dependent pathway. Moreover, Ni et al. also reported that YAP is essential for Tregs differentiation in anti-tumour immunity. Considerable YAP transcription levels are observed in Tregs compared with other subsets of $\mathrm{T}$ cells. In the Treg-specific YAP deficiency mice, the proportions of $\mathrm{CD}^{+}$and $\mathrm{CD} 4^{+} \mathrm{T}$ cells is increased, accompanied with a compromised Treg presence. Mechanismly, deletion of Yap in T cells inhibits Tregs differentiation through limiting TGF $\beta$ B/SMAD and Activin signalling (57). Apart from Tregs, MDSCs comprise another cell population in TME that functions to abolish immune surveillance. YAP activation in tumour cells reduces the secretion of IL-6, which induces the recruitment of MDSCs, resulting in the exhaustion of $\mathrm{CD} 8^{+} \mathrm{T}$ cells, apoptosis of NK cells and dendritic cell maturation disorder. In pancreatic ductal cells with Kras: p53 mutant, YAP activation triggers MDSCs recruitment through binding to the promoter regions of IL-6 and the Csfs (46). In addition to IL-6, recent studies also prove that the Hippo-YAP signalling pathway up-regulates $\mathrm{Cxcl5}$ in prostate tumour, and promoting MDSCs recruitment through the Cxcl5Cxcr2 signalling pathway (55). It remains to be determined, however, whether YAP activation in TAMs influence the above immunosuppressive sells, in light of the evidence indicating that M2 TAMs dramatically facilitate Tregs and MDSCs infiltration $(43,55,58,59)$. Programmed death-1 (PD-1) is a type I transmembrane protein expressed on activated T cells, DC, macrophages, B cells and NK cells, which prevents $\mathrm{T}$ cells activation and inhibits the immune clearance effect of tumour infiltrating lymphocytes. The immune checkpoint protein, programmed death-ligand-1 (PD-L1), is a transmembrane protein expressed on the tumour and immune cell membranes and is the ligand of PD-1. It has been shown that therapeutic antibodies that target PD-L1 or PD-1 are effective in eliminating cancer cells by enhancing the cytotoxicity of $\mathrm{T}$ cells activation $(60,61)$. Recent studies have pointed out that a binding site of TEAD is present around the PD-L1 promoter. YAP can directly bind to the enhancer region of PD-L1 or up-regulate its expression at a transcriptional level in lung melanoma and breast cancer cells (62-64). In addition to PD-L1, CTLA-4 comprises another vital negative regulator of adaptive immune response of $\mathrm{T}$ cells. However, 


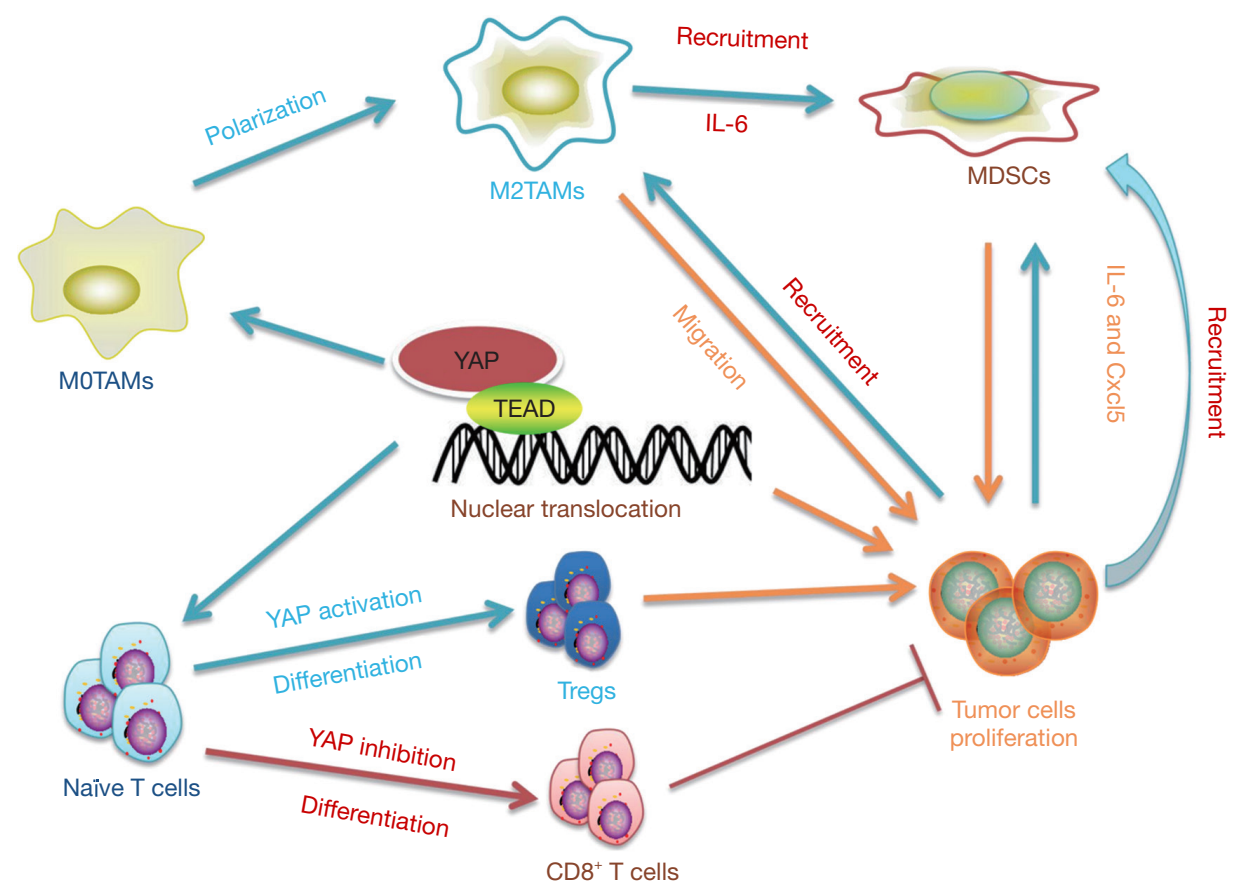

Figure 3 The role of YAP in tumour immunity: Transcription regulator YAP acts a crucial inhibitor in multiple immunological cells. The YAP activation in T cells regulates the differentiation and functions of Tregs and $\mathrm{CD}^{+} \mathrm{T}$ cells, which influences tumour progression further. The YAP activation in TAMs triggers the differentiation and functions of M2 phenotype. YAP expression in tumour cells recruit MDSCs and TAMs via releasing relevant cytokines include IL-6 and Cxc15. TAMs, tumour-associated macrophages; MDSCs, myeloid-derived suppressor cells.

Thaventhiran et al. claimed that the combination of CTLA4 located on T cells and CD80/CD86 expressed on APCs activate Hippo-YAP signalling pathway in T cells, and then the degradation of YAP induced the differentiation of CD 8+ T cells (65). These studies suggest that YAP plays a crucial role in the development of immunological tolerance. Based on the findings of the aforementioned studies, we can conclude that the amplification of YAP expression will weaken the tumour immune response, thus, opening new avenues to regulate tumour immunosuppression.

\section{Conclusions and future prospects}

The infiltration of TAMs in the tumour tissue can be a double edged sword, which functions as an anti-tumour occurrence in patients at the early stages of tumour formation and tends to develop into the M2 phenotype as it progresses to the later stages. Currently, the strategy for anti-tumour therapy against TAMs includes the induction of its apoptosis, repolarization of the M2 TAM to its M1 phenotype, targeting it for an immune checkpoint therapy and adoptive cytotoxic T-cell (CTL) transfer therapy (66-68). Unfortunately, to date, the efficiency of above immunotherapy strategies targeting TAMs has been modest. Although deletion of TAMs in TME attenuated tumour progression noticeably in mouse model, no efficiency was observed in clinical applications $(33,69)$. Based on the above description, it can be determined that YAP not only recruits TAMs to the tumour and its adjacent tissue, but also regulates its polarization directly. Therefore, YAP is a potential target for tumour targeting therapy. To date, the categorical role of the Hippo-YAP pathway in TAMs is still covered. Being the most important target for immunotherapy, the expression of PD-L1 has also been discovered in TAMs $(67,68)$. For instance, in vivo/in vitro experiments, engulfing of exosomes rich in miR-23a-3p secreted by the HCC cells undergoing endoplasmic reticulum stress led to an up-regulation in the expression of PD-L1 in TAMs. However, further studies are still required to investigate the regulation of $\mathrm{PD}-\mathrm{L} 1$ expression in TAMs (70). In addition to the aforementioned functions of the Hippo signalling pathway in TAMs, a study published 
in Science revealed an interesting mechanism underlying the inhibition of tumour progression. Differing from the traditional studies that only focus on the autonomous tumour formation roles of YAP/TAZ, Moya et al. reported that the activation of YAP/TAZ in the peritumoural hepatocytes surrounding the liver tissues markedly suppress tumour growth, thus identifying its multifaceted roles in the regulation of tumour progression. In a syngeneic mouse model, deletion of YAP/TAZ in peritumoural hepatocytes by injecting a Cre-expressing adeno-associated virus 8 (AAV-Cre) into $\mathrm{Yap}^{\mathrm{fl} / f \mathrm{l}}$ and $\mathrm{Taz}^{\mathrm{fl} / \mathrm{fl}}$ double-floxed mice significantly suppressed cholangiocarcinoma cells proliferation and invasion, independent of programmed cell death or feedback inhibition of Hippo signalling pathway. Similar results were also found in hepatocellular carcinoma and melanoma liver metastasis. These interesting results underscore the non-autonomous tumour suppression roles of YAP/TAZ in peritumoural hepatocytes, which may be reminiscent of that of competitive fitness. However, whether the aforementioned mechanisms are present in human organs or other tumours types remains to be determined (71). Therefore, it is of urgently required to further elucidate the regulatory mechanism of YAP in TAMs to improve the efficacy of immunotherapy and ameliorate the outcome of the patients.

\section{Acknowledgments}

Funding: The project was supported by the National Natural Science Foundation of China (81871259, 81530048, 81400650,814700901 , and 81971495), the Foundation of Jiangsu Collaborative Innovation Center of Biomedical Functional Materials, the Priority Academic Program Development of Jiangsu Higher Education Institutions, The Six talent peaks project in Jiangsu Province (2017WSW-019).

\section{Footnote}

Conflicts of Interest: The authors have no conflicts of interest to declare.

Ethical Statement: The authors are accountable for all aspects of the work in ensuring that questions related to the accuracy or integrity of any part of the work are appropriately investigated and resolved.

Open Access Statement: This is an Open Access article distributed in accordance with the Creative Commons Attribution-NonCommercial-NoDerivs 4.0 International License (CC BY-NC-ND 4.0), which permits the noncommercial replication and distribution of the article with the strict proviso that no changes or edits are made and the original work is properly cited (including links to both the formal publication through the relevant DOI and the license). See: https://creativecommons.org/licenses/by-nc-nd/4.0/.

\section{References}

1. Zhou Q, Li L, Zhao B, et al. The hippo pathway in heart development, regeneration, and diseases. Circ Res 2015;116:1431-47.

2. Zheng Y, Pan, D. The Hippo Signaling Pathway in Development and Disease. Developmental Cell 2019;50:264-82.

3. Pu N, Gao S, Yin H, et al. Cell-intrinsic PD-1 promotes proliferation in pancreatic cancer by targeting CYR61/ CTGF via the hippo pathway. Cancer Lett 2019;460:42-53.

4. Shao DD, Xue W, Krall EB, et al. KRAS and YAP1 converge to regulate EMT and tumor survival. Cell 2014;158:171-84.

5. Jia L, Zhang Y, Ji Y, et al. YAP balances the osteogenic and adipogenic differentiation of hPDLSCs in vitro partly through the Wnt/beta-catenin signaling pathway. Biochem Biophys Res Commun 2019;518:154-60.

6. Li Y, Zhou H, Li F, et al. Angiomotin binding-induced activation of Merlin/NF2 in the Hippo pathway. Cell Res 2015;25:801-17.

7. Zhang N, Bai H, David KK, et al. The Merlin/NF2 tumor suppressor functions through the YAP oncoprotein to regulate tissue homeostasis in mammals. Dev Cell 2010;19:27-38.

8. Moon S, Kim W, Kim S, et al. Phosphorylation by NLK inhibits YAP-14-3-3-interactions and induces its nuclear localization. EMBO Rep2017;18:61-71.

9. Zhang B, Shi Y, Gong A, et al. HucMSC ExosomeDelivered 14-3-3zeta Orchestrates Self-Control of the Wnt Response via Modulation of YAP During Cutaneous Regeneration. Stem Cells 2016;34:2485-500.

10. KimY, JhoEH. Regulation of the Hippo signaling pathway by ubiquitin modification. BMB Rep 2018;51:143-50.

11. Wang C, An J, Zhang P, et al. The Nedd4-like ubiquitin E3 ligases target angiomotin/p130 to ubiquitin-dependent degradation. Biochem J 2012;444:279-89.

12. Cheng $\mathrm{Y}, \mathrm{Zhu} \mathrm{Y}, \mathrm{Xu} \mathrm{J}$, et al. $\mathrm{PKN} 2$ in colon cancer cells 
inhibits M2 phenotype polarization of tumor-associated macrophages via regulating DUSP6-Erk1/2 pathway. Mol Cancer 2018;17:13.

13. Yang M, Liu J, Shao J, et al. Cathepsin S-mediated autophagic flux in tumor-associated macrophages accelerate tumor development by promoting M2 polarization. Mol Cancer 2014;13:43.

14. Locati M, Mantovani A, Sica A. Macrophage activation and polarization as an adaptive component of innate immunity. Adv Immunol 2013;120:163-84.

15. Chen Y, Zhang S, Wang Q, et al. Tumor-recruited M2 macrophages promote gastric and breast cancer metastasis via M2 macrophage-secreted CHI3L1 protein. J Hematol Oncol 2017;10:36.

16. Sica A, Larghi P, Mancino A, et al. Macrophage polarization in tumour progression. Semin Cancer Biol 2008;18:349-55.

17. Sica A, Schioppa T, Mantovani A, et al. Tumour-associated macrophages are a distinct $\mathrm{M} 2$ polarised population promoting tumour progression: potential targets of anticancer therapy. Eur J Cancer 2006;42:717-27.

18. Prima V, Kaliberova LN, Kaliberov S, et al. COX2/ mPGES1/PGE2 pathway regulates PD-L1 expression in tumor-associated macrophages and myeloidderived suppressor cells. Proc Natl Acad Sci USA 2017;114:1117-22.

19. Kimbrough-Allah MN, Millena AC, Khan SA. Differential role of PTEN in transforming growth factor beta (TGFbeta) effects on proliferation and migration in prostate cancer cells. Prostate 2018;78:377-89.

20. Jones JD, Sinder BP, Paige D, et al. Trabectedin Reduces Skeletal Prostate Cancer Tumor Size in Association with Effects on M2 Macrophages and Efferocytosis. Neoplasia 2019;21:172-84.

21. Zheng X, Turkowski K, Mora J, et al. Redirecting tumorassociated macrophages to become tumoricidal effectors as a novel strategy for cancer therapy. Oncotarget 2017;8:48436-52.

22. Sica A. Role of tumour-associated macrophages in cancerrelated inflammation. Exp Oncol 2010;32:153-8.

23. Aras S, Zaidi MR. TAMeless traitors: macrophages in cancer progression and metastasis. Br J Cancer 2017;117:1583-91.

24. Pan Z, Tian YQ, Cao CS, et al. The emerging role of Yap/Taz in tumor immunity. Molecular Cancer Research 2019;17:1777-86.

25. Feng $\mathrm{Y}$, Liang $\mathrm{Y}, \mathrm{Zhu} \mathrm{X}$, et al. The signaling protein Wnt5a promotes TGF $\beta 1$-mediated macrophage polarization and kidney fibrosis by inducing the transcriptional regulators Yap/Taz. J Biol Chem 2018;293:19290-302.

26. Liu L, Ge D, Ma L, et al. Interleukin-17 and prostaglandin E2 are involved in formation of an M2 macrophagedominant microenvironment in lung cancer. J Thorac Oncol 2012;7:1091-100.

27. Liang $S, M a$ HY, Zhong $Z$, et al. NADPH Oxidase 1 in Liver Macrophages Promotes Inflammation and Tumor Development in Mice. Gastroenterology 2019;156:1156-72.e6.

28. Wei C, Yang C, Wang S, et al. M2 macrophages confer resistance to 5 -fluorouracil in colorectal cancer through the activation of CCL22/PI3K/AKT signaling. Onco Targets Ther 2019;12:3051-63.

29. Li X, Yao W, Yuan Y, et al. Targeting of tumourinfiltrating macrophages via CCL2/CCR2 signalling as a therapeutic strategy against hepatocellular carcinoma. Gut 2017;66:157-67.

30. Nywening TM, Wang-Gillam A, Sanford DE, et al. Targeting tumour-associated macrophages with CCR2 inhibition in combination with FOLFIRINOX in patients with borderline resectable and locally advanced pancreatic cancer: a single-centre, open-label, dose-finding, nonrandomised, phase 1b trial. Lancet Oncol 2016;17:651-62.

31. Pyonteck SM, Akkari L, Schuhmacher AJ, et al. CSF$1 \mathrm{R}$ inhibition alters macrophage polarization and blocks glioma progression. Nat Med 2013;19:1264-72.

32. Chen XJ, Deng YR, Wang ZC, et al. Hypoxia-induced ZEB1 promotes cervical cancer progression via CCL8dependent tumour-associated macrophage recruitment. Cell Death \& Disease 2019;10:508.

33. Gutmann DH, Helmut K. Microglia/Brain Macrophages as Central Drivers of Brain Tumor Pathobiology. Neuron 2019;104:442-9.

34. Zhou TY, Zhou YL, Qian MJ, et al. Interleukin-6 induced by YAP in hepatocellular carcinoma cells recruits tumorassociated macrophages. J Pharmacol Sci 2018;138:89-95.

35. Weng YS, Tseng HY, Chen YA, et al. MCT-1/miR-34a/ IL-6/IL-6R signaling axis promotes EMT progression, cancer stemness and M2 macrophage polarization in triple-negative breast cancer. Mol Cancer 2019;18:42.

36. Kim W, Khan SK, Liu YC, et al. Hepatic Hippo signaling inhibits protumoural microenvironment to suppress hepatocellular carcinoma. Gut 2018;67:1692-703.

37. Guo X, Zhao Y, Yan H, et al. Single tumor-initiating cells evade immune clearance by recruiting type II macrophages. Genes Dev 2017;31:247-59. 
38. Zhang Q, Chai S, Wang W, et al. Macrophages activate mesenchymal stem cells to acquire cancer-associated fibroblast-like features resulting in gastric epithelial cell lesions and malignant transformation in vitro. Oncol Lett 2019;17:747-56.

39. Calvo F, Ege N, Grande-Garcia A, et al. Mechanotransduction and YAP-dependent matrix remodelling is required for the generation and maintenance of cancer-associated fibroblasts. Nat Cell Biol 2013;15:637-46.

40. Liu F, Lagares D, Choi KM, et al. Mechanosignaling through YAP and TAZ drives fibroblast activation and fibrosis. Am J Physiol Lung Cell Mol Physiol 2015;308:L344-57.

41. Cortes E, Sarper M, Robinson B, et al. GPER is a mechanoregulator of pancreatic stellate cells and the tumor microenvironment. EMBO Rep 2019;20:e46556.

42. Li, X, Liu, R, Su X, et al. Harnessing tumor-associated macrophages as aids for cancer immunotherapy. Mol Cancer 2019;18:177.

43. White SM, Murakami S, Yi C. Murakami and C. Yi, The complex entanglement of Hippo-Yap/Taz signaling in tumor immunity. Oncogene 2019;38:2899-909.

44. Sahraei M, Chaube B, Liu Y, et al. Suppressing miR-21 activity in tumor-associated macrophages promotes an antitumor immune response. J Clin Invest 2019;129:5518-36.

45. Huang YJ, Yang CK, Wei PL, et al. Ovatodiolide suppresses colon tumorigenesis and prevents polarization of M2 tumor-associated macrophages through YAP oncogenic pathways. J Hematol Oncol 2017;10:60.

46. Murakami S, Shahbazian D, Surana R, et al. Yes-associated protein mediates immune reprogramming in pancreatic ductal adenocarcinoma. Oncogene 2017;36:1232-44.

47. Zhang YL, Li Q, Yang XM, et al. SPON2 Promotes M1like Macrophage Recruitment and Inhibits Hepatocellular Carcinoma Metastasis by Distinct Integrin-Rho GTPaseHippo Pathways. Cancer Res 2018;78:2305-17.

48. Zhou Y, Huang T, Zhang J, et al. Emerging roles of Hippo signaling in inflammation and YAP-driven tumor immunity. Cancer Lett 2018;426:73-9.

49. Woo Y, Kim H, Kim KC, et al. Tumor-secreted factors induce IL-1beta maturation via the glucose-mediated synergistic axis of mTOR and NF-kappaB pathways in mouse macrophages. PLoS One 2018;13:e0209653.

50. Xue J, Schmidt SV, Sander J, et al. Transcriptome-based network analysis reveals a spectrum model of human macrophage activation. Immunity 2014;40:274-88.
51. Kaur S, Chang T, Singh SP, et al. CD47 signaling regulates the immunosuppressive activity of VEGF in T cells. J Immunol 2014;193:3914-24.

52. Ansari D, Ohlsson H, Althini C, et al. The Hippo Signaling Pathway in Pancreatic Cancer. Anticancer Res 2019;39:3317-21.

53. Rehrauer $\mathrm{H}, \mathrm{Wu} \mathrm{L}$, Blum W, et al. How asbestos drives the tissue towards tumors: YAP activation, macrophage and mesothelial precursor recruitment, RNA editing, and somatic mutations. Oncogene 2018;37:2645-59.

54. Yamauchi T, Moroishi T. Hippo Pathway in Mammalian Adaptive Immune System. Cells 2019;8:398.

55. Wang G, Lu X, Dey P, et al. Targeting YAP-Dependent MDSC Infiltration Impairs Tumor Progression. Cancer Discov 2016;6:80-95.

56. An Y, Adams JR, Hollern DP, et al. Cdh1 and Pik3ca Mutations Cooperate to Induce Immune-Related Invasive Lobular Carcinoma of the Breast. Cell Rep 2018;25:702-14.e6.

57. Ni X, Tao J, Barbi J, et al. YAP Is Essential for TregMediated Suppression of Antitumor Immunity. Cancer Discov 2018;8:1026-43.

58. Sun W, Wei FQ, Li WJ, et al. A positive-feedback loop between tumour infiltrating activated Treg cells and type 2-skewed macrophages is essential for progression of laryngeal squamous cell carcinoma. Br J Cancer 2017;117:1631-43.

59. Chang Y, Fu XR, Cui M, et al. Activated hippo signal pathway inhibits cell proliferation and promotes apoptosis in NK/T cell lymphoma cells. Cancer Med 2019;8:3892-904.

60. Zamarin D, Ricca JM, Sadekova S, et al. PD-L1 in tumor microenvironment mediates resistance to oncolytic immunotherapy. J Clin Invest 2018;128:1413-28.

61. Vari F, Arpon D, Keane C, et al. Immune evasion via PD-1/PD-L1 on NK cells and monocyte/macrophages is more prominent in Hodgkin lymphoma than DLBCL. Blood 2018;131:1809-19.

62. Kim MH, Kim CG, Kim SK, et al. YAP-Induced PD-L1 Expression Drives Immune Evasion in BRAFi-Resistant Melanoma. Cancer Immunol Res 2018;6:255-66.

63. Miao J, Hsu PC, Yang YL, et al. YAP regulates PDL1 expression in human NSCLC cells. Oncotarget 2017;8:114576-87.

64. Janse van Rensburg HJ, Azad T, Ling M, et al. The Hippo Pathway Component TAZ Promotes Immune Evasion in Human Cancer through PD-L1. Cancer Res 2018;78:1457-70. 
65. Thaventhiran JE, Hoffmann A, Magiera L, et al. Activation of the Hippo pathway by CTLA-4 regulates the expression of Blimp-1 in the CD8+ T cell. Proc Natl Acad Sci USA 2012;109:E2223-9.

66. Guan $\mathrm{W}, \mathrm{Hu} \mathrm{J}$, Yang L, et al. Inhibition of TAMs improves the response to docetaxel in castration-resistant prostate cancer. Endocr Relat Cancer 2019;26:131-40.

67. Etzerodt A, Tsalkitzi K, Maniecki M, et al. Specific targeting of CD163(+) TAMs mobilizes inflammatory monocytes and promotes $\mathrm{T}$ cell-mediated tumor regression. J Exp Med 2019;216:2394-411.

68. Hagn M, Blackwell SE, Beyer T, et al. B-CLL cells acquire APC- and CTL-like phenotypic characteristics

Cite this article as: Yang W, Yang S, Zhang F, Cheng F, Wang X, Rao J. Influence of the Hippo-YAP signalling pathway on tumor associated macrophages (TAMs) and its implications on cancer immunosuppressive microenvironment. Ann Transl Med 2020;8(6):399. doi: 10.21037/atm.2020.02.11 after stimulation with $\mathrm{CpG}$ ODN and IL-21. Int Immunol 2014;26:383-95.

69. Li X, Liu R, Su X, et al. Harnessing tumor-associated macrophages as aids for cancer immunotherapy. Mol Cancer 2019;18:177.

70. Liu J, Fan L, Yu H, et al. Endoplasmic Reticulum Stress Causes Liver Cancer Cells to Release Exosomal miR-23a-3p and Up-regulate Programmed Death Ligand 1 Expression in Macrophages. Hepatology 2019;70:241-58.

71. Moya IM, Castaldo SA, Van DML, et al. Peritumoral activation of the Hippo pathway effectors YAP and TAZ suppresses liver cancer in mice. Science 2019;366:1029-34. 